\title{
Codon 249 P53 Gene Mutation Among Hepatocellular Carcinoma Patients in Western Kenya
}

\author{
Colins 0 Oduma ${ }^{1 *}$, Bartholomew N Ondigo ${ }^{1,3}$ and James H Kimotho ${ }^{2}$ \\ ${ }^{1}$ Department of Biochemistry and Molecular Biology, Egerton University, Nakuru, Kenya \\ ${ }^{2}$ Kenya Medical Research Institute, Nairobi, Kenya \\ ${ }^{3}$ Kenya Medical Research Institute, Kisumu, Kenya
}

\begin{abstract}
Background: Alteration(s) on p53 gene adversely affects their regulatory functions resulting to cancers. To our knowledge, no previous studies have analyzed mutation in codon 249 of $p 53$ gene in hepatocellular carcinoma (HCC) patients in Kenyan population. In our study, we investigated for these mutations in HCC patients presenting with stage one cancer in the Kenyan population.
\end{abstract}

Methods: A total of forty-six (46) stage one HCC patients and ten (10) controls were prospectively recruited from Moi Teaching and Referral Hospital (MTRH), Kenya. DNA was extracted and purified from 200 uL aliquots of plasma and PCR amplified then sequenced using p53 exon 7 forward and reverse primers. Codon 249 p53 gene mutation detection and analysis were done using Molecular Evolutionary Genetics Analysis v.6.0, Genetyx v.9.0 and Integrative Genome Viewer v. 2.4.4. The male to female ratio for both patients and controls was 1:1. The age range for HCC patients was from 25 to 67 years with a median of 42 years, and from 24 to 64 years with a median of 41 years for the controls.

Results: Guanine (G) - to - thymine (T) transversion in the third base of codon 249 of p53 gene was detected in 8 of the 46 HCC patients (17.39\%) and 1 of the 10 controls (10\%). There was no significant difference across gender among HCC subjects with and without mutation $(\mathrm{p}=0.4549)$ at $5 \%$ level of significance. There was a striking picture of significant existence of such mutation in a much older population in both the HCC patients $(\mathrm{p}=<.0001)$ and the controls $(p=0.0003)$ at $5 \%$ level of significance, suggesting that being in the old age is more susceptible to such mutation. There was no significant statistical association of codon 249 mutation with HCC ( $p=0.6821)$ at $5 \%$ level of significance.

Conclusion: There is no association between $p 53$ exon 7 codon 249 mutations and hepatocellular carcinoma from patients presenting with stage I cancer. There is need for supplemental cross-sectional large sample sizes studies of HCC patients presenting with different stages to observe pattern across stages.

KEYWORDS: Codon 249; Exon 7; Gene mutation; Hepatocellular carcinoma; p53 Stage one

ABBREVIATIONS: HCC: Hepatocellular Carcinoma; MTRH: Moi Teaching and Referral Hospital; DNA: Deoxyribonucleic acid; PCR: Polymerase Chain Reaction; G: Guanine; T: Thymine; KEMRI: Kenya Medical Research Institute; EDTA: Ethylenediaminetetraacetic acid; BLAST: Basic Local Alignment Search Tool; NCBI: National Center for Biotechnology Information; MEGA: Molecular Evolutionary Genetics Analysis; IARC: International Agency for Research on cancer; AFB1: Aflatoxin B1; HBV: Hepatitis B virus; HCV: Hepatitis C virus

Quick Response Code:

Address for correspondence: Colins O Oduma, Department of Biochemistry and Molecular Biology, Egerton University, Nakuru, Kenya; Email: odumaccc@gmail.com Received: January 21, 2020 Acceptance: January 27, 2020 Published: January 31, 2020 How to cite this article: Colins OO, Bartholomew NO, James HK. Codon 249 P53 Gene Mutation Among Hepatocellular Carcinoma Patients in Western Kenya. 2020 - 2(1) OAJBS. ID.000136. DOI: 10.38125/OAJBS.000136 


\section{INTRODUCTION}

Hepatocellular carcinoma (HCC) is one of the most common malignancies worldwide. It is increasing in incidence and has a very high fatality rate of 800,000 deaths globally per annum [1]. According to World Health Organization [2], it is the fourth leading cause of cancer-related death in the world and fifth in Africa. The HCC cases constitute the fifth most common in men globally, and seventh among women, with over 600,000 new cases diagnosed annually worldwide [1]. According to Global Burden of Disease Liver Cancer Collaboration 2015, incidence of HCC varies broadly worldwide between geographical regions, with some of the highest incidence rates found in the West, Asia and Africa. Noteworthy, there is dearth of information on the overall incidences of HCC in Kenyan population. This is occasioned by lack of national repository for cancer cases and the few available setting-based cancer registries.

P53 gene play crucial role in tumor suppression. The gene hampers progression of cell cycle if the DNA is damaged. This it does through (i) preventing the replication of damaged DNA while activating the transcription of proteins taking part in DNA repair and (ii) triggering the cell to induce apoptosis, cell cycle arrest or senescence in the event the damage is severe and cannot be repaired $[3,4]$. Alteration in $p 53$ gene affect major regulators of various signaling pathways involved in tumor suppression. The alterations are established to be observed in most cancers $[5,6]$, and are majorly as a result of inactivation of $p 53$ gene by mutation. The $p 53$ mutations are distributed in all coding exons, with a strong predominance in exons 4-9, encoding the DNA-binding domain of the protein [7]. Studies have demonstrated that mutant $p 53$ contribute immensely to replication of damaged DNA and successive tumor progression. The mutant proteins bind to $p 53$ response elements thereby weakening the process of DNA repair and $p 53$-mediated apoptosis $[8,9]$. Differences in ethnicity and geographical location among other factors have varied impact on $p 53$ mutation profiles $[5,10]$. To our knowledge, no study has analyzed mutation in codon 249 of $p 53$ gene in hepatocellular carcinoma (HCC) patients in Kenyan population. In the present study, we screened for the presence of mutation in HCC patients presenting with stage one cancer, the initial stage of cancer presentation, in Moi Teaching and Referral Hospital (MTRH), Kenya.

\section{METHODS}

\section{Study Site and Study Population}

The study population comprised hepatocellular carcinoma (HCC) patients who presented with stage one cancer at Moi Teaching and Referral Hospital (MTRH), Kenya. A patient with HCC was defined as having liver cancer based on the patient's medical record and cancer registry file. All the patients were diagnosed with HCC by histopathological diagnosis. Medical profile of the patients including age, ethnicity, gender and residential area were obtained from patient's medical record and cancer registry file. None of the patients had received any cancer treatment by the time of sample collection. The MTRH was selected as it is one of the largest national referral hospitals in Western Kenya, where rates of HCC risk factors are high [11] and its technical capabilities for detection and characterization of carcinomas at its histopathology laboratory. The HCC cases were purposively selected from hospital records between February-June 2017. A total of forty-six (46) HCC cases were selected and a further ten (10) healthy individuals were also included for the study. Healthy controls were clinically defined as individuals who had tested negative for HCC serological molecular marker- alpha fetoprotein level. The ratio of male to female was 1:1 for both HCC patients and controls. The age range for HCC patients was from 25 to 67 years with a median of 42 years, and from 24 to 64 with a median of 41 years for the controls.

\section{Ethical Consideration}

Ethical approval to conduct the study was sought and obtained from the Kenya Medical Research Institute Scientific Ethics Review Unit (KEMRI/SERU/CVR/001/3211) and Eldoret Cancer Registry (ECR/DRA/2017/001). Further, the participants consented for the study prior to blood draw and obtaining medical records.

\section{Collection and Preparation of Blood Samples}

Blood samples were collected in vails anti-coagulated with EDTA and separated into plasma at MTRH thereafter shipped on dry ice to KEMRI Production Unit molecular laboratories. The plasma samples were then stored in aliquots at $-80^{\circ} \mathrm{C}$ in ultra-low temperature freezer (Eppendorf AG Hamburg) until subsequent testing.

\section{Extraction of DNA from Plasma}

Circulating DNA of Human p53 tumor suppressor gene was extracted from $200 \mu \mathrm{l}$ of plasma samples using QI Amp DNA mini-extraction kit (Qiagen Inc, USA) according to manufacturer's instructions with slight modifications. The extracted DNA was subsequently eluted in $60 \mu \mathrm{l}$ of $\mathrm{AE}$ buffer and quantified by Nano Drop spectrophotometer (Thermo Fisher Scientific) and stored at $-30^{\circ} \mathrm{C}$ in biomedical freezer (Panasonic, Japan) until tested.

\section{PCR Amplification of Human p53 exon 7}

Amplification of the targeted exon 7 was undertaken in a single stage $25 \mu \mathrm{l}$ reaction mix composed of $2.5 \mu \mathrm{l}$ of genomic DNA template, $2.5 \mu$ of 10X PCR buffer (10 mMTris-HCL, pH 9.0, 50mM KCL, $1.5 \mathrm{mM}$ $\mathrm{MgCl}_{2}, 0.01 \%$ gelatin and $0.1 \%$ triton X-100), $1 \mu \mathrm{l}$ of dNTP mix, $2 \mu \mathrm{l}$ of $25 \mathrm{mM}$ stock $\mathrm{MgCl}_{2}, 0.2 \mu \mathrm{l}$ of $5 \mathrm{U}$ of Taq DNA polymerase (Qiagen Inc, USA), $0.5 \mu \mathrm{l}$ each of a $20 \mathrm{uM}$ stock of forward primer and reverse primer of sequences (5'-CTTGCCACAGGTCTCCCCAA-3') and (5'AGGGGTCAGCGGCAAGCAGA-3') respectively and amplified in a Veritti-96-well thermal cycler (Applied Biosystems, Foster city, USA) for 35 cycles. Each cycle entailed initial denaturation at $95^{\circ} \mathrm{C}$ for 10 minutes, denaturation at $95^{\circ} \mathrm{C}$ for 45 seconds, primer annealing at $58^{\circ} \mathrm{C}$ for 30 seconds and extension at $72^{\circ} \mathrm{C}$ for 7 minutes. After that, a $4 \mu \mathrm{l}$ aliquot of PCR product was electrophoresed by using $1 \%$ agarose (Fisher Scientific), $2 \mu \mathrm{l}$ of $5 \mathrm{X}$ Gelpilot DNA loading Dye (Qiagen Inc, USA) together with 100 bp Tracklt DNA ladder (Invitrogen, California, US) in 1X TBE buffer containing SYBR-safe DNA gel stain (Invitrogen, California, US) and visualized using an ultraviolet trans-illuminator gel Doc-It2 Imager then viewed using Vision Works LS software v.7.1. The expected band size of the PCR product was 249 base pairs (bp).

\section{DNA Sequencing}

All PCR-positive amplicons were purified using Qiagen Gel purification kit according to manufacturers recommended protocol. The purified DNA was quantified by Nano Drop spectrophotometer (Thermo Fisher Scientific) and purified DNA (50 ng) sent for sequencing at Macrogen, Inc. (Netherlands) using forward primer (5'-CTTGCCACAGGTCTCCCCAA-3') and reverse primer (5'-AGGGGTCAGCGGCAAGCAGA-3') according to Macorgen Inc. guidelines. 


\section{Mutation Detection and Analysis}

The sequences were aligned to the $p 53$ gene sequences by uploading the sequences onto National Center for Biotechnology Information (NCBI) - Basic Local Alignment Search Tool (BLAST) program (www.ncbi.org) for identity confirmation. Mutations on the sequences were analyzed using Molecular Evolutionary Genetics Analysis (MEGA) v.6.0 software bioinformatics editing tool. Consensuses of both forward and reverse sequences were assembled and built using GENETYX software version 9.0 that finds homology and create contigs. The contigs were then aligned to the $p 53$ gene reference sequence from International Agency for Research on Cancer (IARC) database using ClustalW alignment tool on MEGA v.6.0. The output of the aligned sequences and the reference sequence was then viewed using MEGA v.6.0. The aligned sequences were then uploaded into T-coffee and subsequently submitted to ESPript v.3.0 software for analysis.

\section{Statistical Analysis}

Tests for statistical significance of mutation profile were done using the chi-square test and Fisher's exact test. To examine possible association between mutations in p53 exon 7 and hepatocellular carcinoma, we analyzed $2 \times 2$ table using Fisher's exact test in SAS version 9.4. P-value less than 0.05 was considered statistically significant.

\section{RESULTS}

Study participants demographic characteristics A total of fortysix (46) stage I HCC subjects and ten (10) control subjects were considered as shown in Table 1. The ages for HCC cases and controls were within the bracket of significant risk for HCC development. This included ages 20-33 for low risk, 34-49 for medium risk and $\geq 50$ for high risk as shown in Table 1 . The ratio of male to female was 1:1 for both HCC subjects and control subjects. The age range for HCC subjects was from 25 to 67 years, and from 24 to 64 years for the controls. The median and mean ages for HCC subjects (42 and 44.35 respectively) and the control subjects (41 and 42.7 respectively) were not equal.

Table 1: Demographic characteristics of study participants.

\begin{tabular}{|c|c|c|}
\hline & HCC & Control \\
\hline Median age (years) & 42 & 41 \\
\hline Age groups in years & & \\
\hline $20-33$ & 8 & 5 \\
\hline $34-49$ & 23 & 3 \\
\hline$\geq 50$ years & 15 & $1: 01$ \\
\hline
\end{tabular}

\section{Mutation Profile in Hepatocellular Carcinoma (HCC) and Control Subjects}

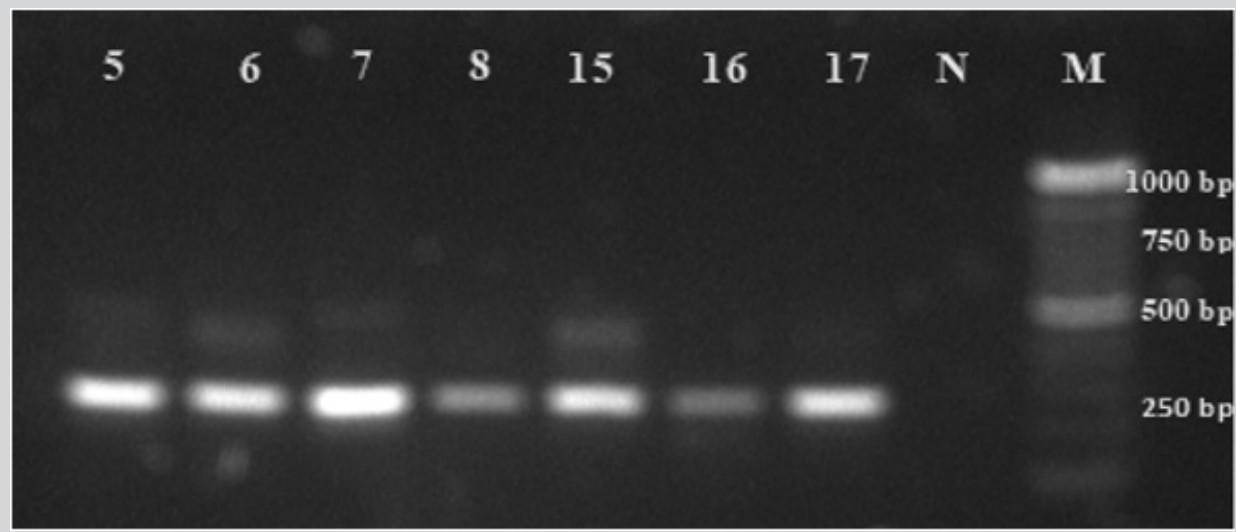

Figure 1: SYBR-safe DNA gel electrophoresis image of amplified products of exon 7 of p53 gene.

Table 2: Number and proportion of individuals with hepatocellular carcinoma with and without mutation at codon 249 by gender and age groups.

\begin{tabular}{|c|c|c|c|}
\hline Characteristic & Presence of Mutation, $\mathbf{n}(\%)$ & Absence of Mutation n (\%) & $P$ value \\
\hline \multicolumn{4}{|l|}{ Gender } \\
\hline Male & $5(21.7)$ & $18(78.3)$ & \multirow{2}{*}{0.4549} \\
\hline Female & $3(13.0)$ & $20(87.0)$ & \\
\hline \multicolumn{4}{|c|}{ Age group } \\
\hline $20-33$ & $0(0)$ & $8(21.1)$ & \multirow{3}{*}{$<.0001^{\mathrm{a}}$} \\
\hline $34-49$ & $1(12.5)$ & $22(57.9)$ & \\
\hline$\geq 50$ & $7(87.5)$ & $8(21.1)$ & \\
\hline
\end{tabular}

Specific mutation: Guanine (G)-to-thymine (T) transversion in the third base of codon 249 of p53 gene corresponding to arginine- to -serine amino acid substitution. aStatistical significance determined by Chi-square Test. ${ }^{*} p<0.05$ was considered statistically significant. All values in bold are statistically significant at $p<0.05$. 
Polymerase chain reaction amplicons for exon 7 of $p 53$ gene were visualized using ultraviolet trans-illuminator gel Doc-It2 Imager using Vision Works LS software as shown in Figure 1. Nucleotide sequences alignment and protein sequences alignment of codon 249 of $p 53$ gene in HCC patients and controls are shown in Figure 2. A selective guanine (G)-to-thymine (T) transversion mutation in the third base of codon 249 of $p 53$ gene, corresponding to arginine-to-serine amino acid substitution at codon codon 249 was present in eight (8) of the forty six patients with HCC (Table 2). Among the HCC patients with the mutation, there were more males than females (Table 2). There was no significant difference across gender among HCC patients with and without the selective mutation $(p=0.4549)$ at $5 \%$ level of significance. However, there was statistically significant difference in median age among HCC patients with and without the selective mutation $(\mathrm{p}=0.0010)$. There was also a striking picture of significant existence of codon 249 mutation in a much older population in the HCC patients $(\mathrm{p}<.0001)$ at $5 \%$ level of significance (Table 2).

Lane 5 represent amplified product of a positive control. Lanes 6, 7 and 8 represent amplified products of exon 7 from hepatocellular carcinoma samples. Lanes 15, 16 and 17 represent amplified products of exon 7 from control subject samples. Lane $\mathrm{N}$ is a negative control for PCR. Lane $\mathrm{M}$ is a $1 \mathrm{~kb}$ DNA size molecular marker for amplification. Molecular grade water was used as negative control for the PCR amplification. The bands (249 bp) show positive amplification of exon 7 of $p 53$ gene (Figure 1).

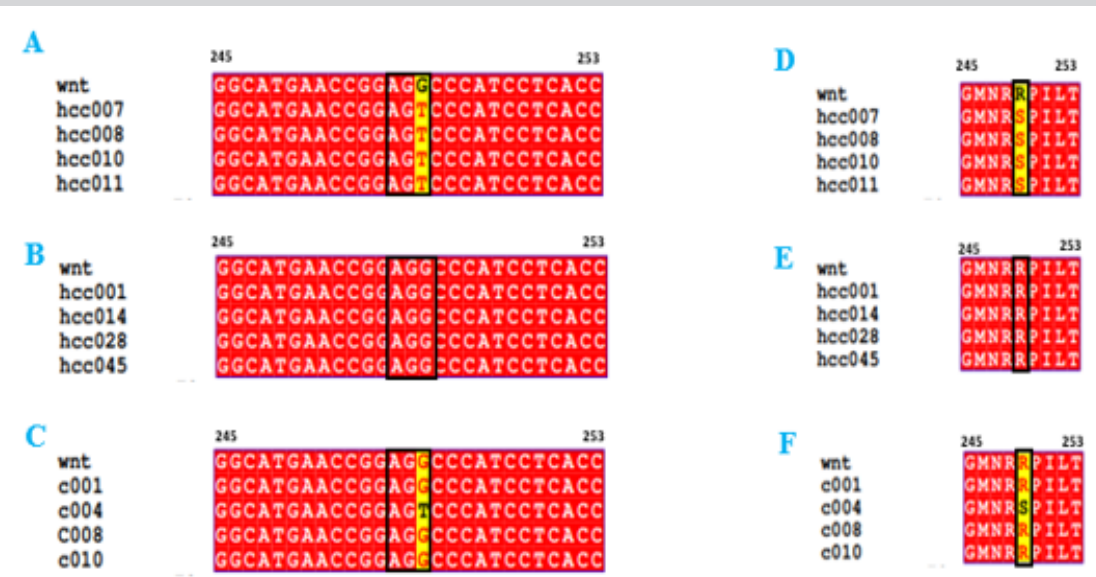

Figure 2: Multiple sequences alignment of exon 7 of p53 gene (codons 245-253).

Panel A. Multiple nucleotide sequences alignment of hepatocellular carcinoma cases with mutation at codon 249 (boxed) represented by nucleotide base $\mathrm{T}$ (yellow background). Panel B. Multiple nucleotide sequences alignment of hepatocellular carcinoma cases without mutation at codon 249 (boxed). Panel C. Multiple sequences alignment of controls. Mutation in one of the controls at codon 249 (boxed) is represented by nucleotide base $\mathrm{T}$ (yellow background). Panel D. Multiple protein sequences alignment of hepatocellular carcinoma cases with mutation at codon 249 (boxed) represented by amino acid residue S (yellow background). Panel E. Multiple protein sequences alignment of hepatocellular carcinoma cases without mutation at codon 249 (boxed). Panel F. Multiple protein sequences alignment of controls. Mutation in one of the controls at codon 249 (boxed) is represented by $\mathrm{S}$ (yellow background). Panels A-C, T: Thymine Nucleotide; G: Guanine Nucleotide; C: Cytosine Nucleotide; A: Adenine Nucleotide. Panels D-F; G: Glycine Residue; M: Methionine Residue; N: Asparagine Residue; R: Arginine Residue; P: Proline Residue; I: Isoleucine Residue; L: Leucine Residue; T: Threonine Residue; S: Serine. wnt: Wild Type (Figure 2).

Table 3: Number and proportion of control individuals with and without mutation at codon 249 by gender and age group.

\begin{tabular}{|c|c|c|c|}
\hline Characteristic & Presence of Mutation, $\mathrm{n}(\%)$ & Absence of Mutation $\mathbf{n}(\%)$ & $P$ value \\
\hline \multicolumn{4}{|l|}{ Gender } \\
\hline Male & $1(20.0)$ & $4(80.0)$ & \multirow{2}{*}{0.4549} \\
\hline Female & $0(0)$ & $5(100.0)$ & \\
\hline \multicolumn{4}{|c|}{ Age group } \\
\hline $20-33$ & $0(0)$ & $2(22.2)$ & \multirow{3}{*}{$<.0001^{\mathrm{a}}$} \\
\hline $34-49$ & $0(0)$ & $5(55.6)$ & \\
\hline$\geq 50$ & $1(100.0)$ & $2(22.2)$ & \\
\hline
\end{tabular}

Specific mutation: Guanine (G)-to-thymine (T) transversion in the third base of codon 249 of p53 gene corresponding to arginine-to-serine substitution. aStatistical significance determined by Chi-square Test. * $p<0.05$ was considered statistically significant. All values in bold are statistically significant at $p<0.05$. 
A selective guanine (G)-to-thymine $(\mathrm{T})$ transversion mutation in the third base of codon 249 of p53 gene; corresponding to arginine - to -serine amino acid substitution at codon 249 was present in one of the ten control subjects (Table 3). Also notable was significant existence of such mutation in a much older population in the controls $(p=0.0003)$ at $5 \%$ level of significance. The difference across gender was however statistically significant among the controls with and without codon 249 mutation $(\mathrm{p}=<.0001)$ at level of significance $p<0.05$. There was statistically insignificant difference for median age among the control $(p=0.3851)$ at level of significance $\mathrm{p}<0.05$ (Table 3).

\section{Codon 249 Mutation and HCC}

The findings showed no statistically significant association of codon 249 mutation and HCC ( $\mathrm{p}=0.6821)$.

\section{DISCUSSION}

In this study; we first sought to identify the profile of $p 53$ gene exon 7 codon 249 gene mutation(s) among HCC patients presenting with stage I cancer disease. To the best of our knowledge; we report the first picture of $p 53$ exon 7 codon 249 mutation profile in HCC patients in Kenyan population. This study found a selective guanine (G) - to - thymine (T) transversion mutation in the third base of codon 249 in DNA isolated from HCC patients. This mutation corresponds to arginine-to-serine substitution at the codon $249 \mathrm{of}$ the $p 53$ gene. Our findings corroborate limited data available among Guangxi; Taiwan and Gambian populations where similar mutations were reported [12-14]

Males were overrepresented in the mutation positive categories in HCC subjects. However; there was no significant difference across gender among hepatocellular carcinoma subjects with and without mutation ( $\mathrm{p}=0.4549$ ) at $5 \%$ level of significance. This could be attributed to a possible occurrence of faster and more severe hepatocellular carcinogenesis in males than female's Li 2016. Also noticeable is the trend in age among those with the mutation. There was a striking picture of significant existence of such mutation in a much older population $(\mathrm{p}=<.0001)$ at $5 \%$ level of significance; implying that order individuals were more susceptible to mutation compared to the younger individuals. A possible explanation would be that; according to National Cancer Institute 2017; occurrence of mutation is leveraged on long duration of living characterized by excessive exposure to mutagens and carcinogens since the process of mutation occurrence is a gradual process [15]. The prolonged duration of excessive exposure to mutagenic and carcinogenic agents due to aging result in accumulative DNA damage in cells [16]

Mutations in DNA repair genes could be a prerequisite of tumor occurrence or could arise due to random accumulation of mutations during cycling of cancer cells Torgovnick \& Schumacher 2015 Existence of incorrect DNA repair in tumor cells predispose them to accumulate further genetic alterations [17]. Consequently; it is argued that presence of a single mutation alone in DNA is unlikely to cause cancer; rather accumulative or multiple mutations in p53 tumor suppressor gene characterized by long life span [18].

Mutation in $p 53$ gene may lead to expression of a mutant $p 53$ protein that has lost wild type function and may deploy a dominant negative regulation over the rest of wild type $p 53$ that suppress tumor through hetero-oligomerizing with wild-type $p 53$; thus acting as a competitive inhibitor of wild type p53 [7]. The consequence of this is loss of transcriptional activity of wild type $p 53$; a phenomenon referred to as loss of function [19]. Mutations in $p 53$ is mostly followed by loss of heterozygosity during cancer progression implying that the dominant negative regulation activity of mutant $p 53$ is not sufficient to completely inactivate wildtype p53; nevertheless; the dominant regulation activity may in rare events present in late stage of cancer $[7,20]$.

Conversely; the mutant p53 may also well acquire a new tumor-promoting activity that is independent of wild-type $p 53$ through production of oncoproteins that offer cancer cells growth and survival edges; phenomenon referred to as gain of function (GOF) [21]. Mutant $p 53 \mathrm{GOF}$ activities, the most wedging oncogenic activity of $p 53$ protein, impact several aspects of cellular biology including affect chromatin structure ; genomic stability; and activate transcription programs wedging cancer cell metabolism; proteasome activity and microRNA biogenesis [22,23]. These activities may in turn foster mutant $p 53$ stability through increasing glucose levels and crosstalk with other oncogenic pathways; thereby conferring selective advantages for tumor growth and aggressiveness [24].

Existence of guanine to thymine transversion mutation; corresponding to arginine-to-serine substitution; at the codon 249 leads to $p 53$ protein GOF activity in HCC [25]. It is still an open question how mutant $p 53$ GOF activities are enabled by the unique blend of HCC related cues. Nevertheless; it is suggested that the p53 protein GOF activity is achieved through CDK4/cyclin D1-Pin1$p 53-R 249 S-c-M y c$ signaling pathway $[22,26]$. Residues in the $p 53$ protein are critical to its DNA binding function of tumor suppression realized through activation of DNA repair proteins when DNA has sustained damage; inducing growth arrest by holding the cell cycle at the G1/S regulation point on DNA damage recognition; or initiating apoptosis if DNA damage is severe and proves to be irreparable $[27,28]$. The change in amino acid arginine to serine substitution at codon 249 result in non-conservative mutation that affects $p 53$ biochemical properties or pathways through interactions with other proteins involved in cell signaling including Pin1; c-Myc; CDK4 proteins [13,14,22]. Furthermore; the nonconservative mutation may lead to disruption of hydrogen bonding causing incorrect folding of p53 protein; altering transactivation of p53 targets in tumours $[16,26,29]$.

The Pin1; a phosphorylation-dependent prolyl-isomerase is an oncoprotein highly expressed in human cancer responsible for conversion of inactive proteins into active oncoproteins such as $p 53$ mutants or c-Myc $[30,31]$. The protein binds to a phosphorylated resultant serine motif and through this; the protein is suggested to modulate multiple cellular events; including gene regulation; RNA processing; cell proliferation; nuclear import; and differentiation thus enhancing cell proliferation; survival and tumorigenesis [22]. Codon 249 mutation result in the substitution of arginine residue with serine residue; which offers platform for Pin1 phosphorylation activity to induce HCC tumorigenesis [30]. The c-Myc; a nuclear transcription factor; highly expressed in majority of human cancers is important in regulation of gene transcription required in cell proliferation; survival and tumorigenesis; with the overall aim being activation of expression of genes involved in ribosomal biogenesis and protein synthesis [8]. This oncoprotein is essential for growth and progression of HCC [22]. The CDK4 protein is of importance in the G1/S phase of the cell cycle by forming a complex with cyclin D; which is utilized by tumor cells for their survival and growth advantages [32]. The CDK4/cyclin D1 complex phosphorylate p53 mutant protein to inhibit cell senescence and apoptosis hence can perpetuate tumorigenic events; including hepatocellular carcinogenesis [33]. 
Various risk factors associated with HCC development such as AFB1 and HBV perpetuate the GOF activity of $p 53$ protein by creating microenvironment or molecular environment and reprograms oncogenic signaling pathways leading to a rare posttranslational modifications; such as methylation at K370 by SETDB1 [34]; and phosphorylation at codon 249 by CDK4/cyclin D1 [7]. The $p 53$ inactivation due to mutation occur at late stages of cancer; that is characterized by shortening of telomeres and loss of short arm of chromosome 17 (which contains $p 53$ gene) rather than early stage. This partially explain the absence of mutation for the thirty-eight HCC cases who were all stage one HCC subjects. Still; the presence of mutation does not unequivocally indicate that $p 53$ is fully inactive; similarly; the absence of mutation does not necessarily indicate that p53 is functionally proficient [35]. Thus; assessing functional activity of $p 53$ mutants is essential for an accurate indication of clinical relevance. Secondly; we also sought to determine association of $p 53$ gene exon 7 codon 249 mutation(s) with hepatocellular carcinoma. Our study findings showed no statistically significant association of codon 249 mutation with hepatocellular carcinoma $(\mathrm{p}=0.6821)$ at level of significance $p<0.05$. These findings agree with finite data available in Taiwan; United States; Japan; Australia; Gambian and Guangxi populations $[12,13,36]$. Counter-intuitively; the specific codon 249 mutation was not only observed among HCC patients but also in one of the controls. This corroborates earlier findings by Kirk et al. [37] that reported codon 249 mutation presence in 3 of 53 control subjects; and that by Ozturk [38] that reported codon 249 mutation in non-malignant liver tissues. Among p53 mutations signatures for HCC compiled in the International Agency for Research on cancer 2018 p53 mutation database; 66\% occur in patients with HCC originating from regions with a high incidence of HCC and high exposure to dietary AFB1 and excessive tobacco use [12-14]. Additionally; in HCC; a unique transversion at codon 249 (G:C.T:A) is highly prevalent in geographic areas in which the mycotoxin aflatoxin is a widespread contaminant of the food mostly in parts of Africa; Eastern Asia; South America [39]. The excessive AFB1 exposure may induce formation of AFB1-N7-guanine adduct that is implicated in HCC manifestation Mah 2011. Moreover; AFB1 metabolites from cytochrome P450 in the liver causes DNA damage by forming covalent and promutagenic DNA adducts that could cause $\mathrm{G}$ to $\mathrm{T}$ transversion at codon 249 of the $p 53$ gene [22]. This study; however; neither perform aflatoxin exposure level test nor have data on tobacco consumption for the subjects to corroborate this. This study acknowledge that the samples were from patients presenting with only stage one HCC thus the small sample size.

\section{CONCLUSION}

The study did not find any association between mutations in exon 7 codon 249 and stage one HCC. Hence; mutations in exon 7 codon 249 is a poor indicator for prognosis among HCC patients presenting with stage one. There is need for supplementary crosssectional studies on HCC patients of larger sample sizes and on those presenting with different stages to observe pattern across the stages. Also; there is need to look at other exons of $p 53$ gene for possible mutations among HCC patients.

\section{ACKNOWLEDGMENT}

We acknowledge the study participants and the staff of Moi Teaching and Referral Hospital.

\section{GRANT INFORMATION}

This work was supported by KEMRI IRG grant from Kenya Medical Research Institute; protocol Number KEMRI/SERU/
CVR/001/3211 and IRG Number L057. The funders had no role in study design; data collection and analysis; decision to publish; or preparation of the manuscript.

\section{REFERENCES}

1. Stewart SL, Kwong SL, Bowlus CL, Nguyen TT, Maxwell AE, et al. (2016) Racial/ethnic disparities in hepatocellular carcinoma treatment and survival in California, World J Gastroenterol 22(38): 1988-2012.

2. World Health Report. Cancer statistics (2016) Geneva, Switzerland.

3. Kruiswijk F, Labuschagne CF, Vousden KH (2015) p53 in survival, death and metabolic health: A lifeguard with a licence to kill. Nat Rev Mol Cell Biol 16(7): 393-405.

4. Sasaki M, Kawahara K, Nishio M, Mimori K, Kogo R, et al. (2011) Regulation of the MDM2-P53 pathway and tumour growth by PICT1 via nucleolar RPL11. Nature Med 17: 944-951.

5. Kandoth C, McLellan MD, Vandin F, Ye K, Niu B, et al. (2013) Mutational landscape and significance across 12 major cancer types. Nature 502(7471): 333-339.

6. Levine AJ, Oren M (2009) The first 30 years of $p 53$ : growing ever more complex. Nat Rev Cancer 9(10): 749-758.

7. Rivlin N, Brosh R, Oren M, Rotter V (2011) Mutations in the $p 53$ tumor suppressor gene: Important milestones at the various steps of tumourigenesis. Genes Cancer 2(4): 466-474.

8. Luis A, Pierre JH, Crystal T, Javes JM (2012) E2F7, a novel target is upregulated by $p 53$ and mediates DNA damage-dependent transcriptional repression. Genes Dev 26: 1533-1545.

9. Maiuri MC, Galluzzi L, Morselli E, Kepp O, Malik SA (2010) Autophagy regulation by $p 53$. Curr Opin Cell Biol 22(2): 181-185.

10. Wen X, Lu F, Liu S (2016) Prognostic value of $p 53$ mutation for poor outcome of Asian primary liver cancer patients: Evidence from a cohort study and meta-analysis of 988 patients. Onco Targets and Therapy 9: 7425-7433.

11. Ochwoto M, Kimotho JH, Oyugi J, Okoth F, Kioko H, et al. (2016) Hepatitis $\mathrm{B}$ infection is highly prevalent among patients presenting with jaundice in Kenya. BMC Infectious Diseases 16: 101.

12. Mah Y, Hsu C, Liu C, Liu C, Lai M, et al. (2011) Serum p53 gene polymorphisms and severity of hepatitis B or C-related chronic liver diseases in Taiwan. Hapatol Int 5: 814-821.

13. Özdemir FT, Tiftikci A, Sancak S, Eren F, Tahan V, et al. (2010) The Prevalence of the mutation in codon 249 of the $P 53$ gene in patients with hepatocellular carcinoma (HCC) in Turkey. J Gastrointest Cancer 41(3): 185-189.

14. Aldona K, Agnieszka A, Wieslawa P, Arkadiusz C (2009) P53 immunocytochemistry and TP53 gene mutations in patients with chronic hepatitis C virus infection. Cytobiologica, 47(1), 35-42.

15. Janevska D, Chaloska-Ivanova V, Janevski V (2015) Hepatocellular carcinoma: Risk factors, diagnosis and treatment. Open Access Maced J Med Sci 3(4): 732-736.

16. Rivlin N, Koifman G, Rotter V (2013) p53 orchestrates between normal differentiation and cancer. Semin Cancer Biol 32: 10-17.

17. Wright WD, Shah SS, Heyer W (2018) Homologous recombination and the repair of DNA double-strand breaks. J Biol Chem 293(27): 1052410535.

18. Adjiri A (2017) DNA mutations may not be the cause of cancer. Oncol Ther 5(1): 85-101.

19. Wang X, Sun Q (2016) TP53 mutations, expression and interaction networks in human cancers. Oncotarget 8(1): 624-643.

20. Kew MC (2014) Hepatocellular carcinoma: epidemiology and risk factors. Hep Carcioma 4(9): 34-43.

21. Alexandrova LB, Nik-Zainal S, Wedge DC, Campbell PJ, Stratton MR (2013) Deciphering signatures of mutational processes operative in human cancer. Cell Reproduction 3(1): 246-259. 
22. Wang H, Liao P, Zeng XS, Lu H (2019) It takes a team: A gain of function story of $p 53-\mathrm{R} 249 \mathrm{~S}$. J Mol Cell Biol 86: 24-30.

23. Naccarati A, Polakova V, Pardini B, Vodickova L, Hemminki K, et al. (2012). Mutations and polymorphisms in TP53 gene: an overview on the role in colorectal cancer. Mutagenesis 27(2): 211-218.

24. Mantovani F, Walerych D, Sal GD (2017) Targeting mutant $p 53$ in cancer: a long road to precision therapy. FEBS J 284(6): 837-850.

25. Liao P, Zeng SX, Zhou X, Chen T, Zhou F, et al. (2017) Mutant $p 53$ Gains Its Function via c-Myc Activation upon CDK4 Phosphorylation at Serine 249 and Consequent PIN1 Binding. Mol Cell 68(6): 1134-1146.

26. Muller PAJ, Vousden KH (2014) Mutant $p 53$ in cancer: New functions and therapeutic opportunities. Cancer Cell 25(3): 304-317.

27. Nasr Z, Pelletier J (2012) Tumour progression and metastasis: Role of translational deregulation. Anticancer Res 32(8): 3077-3084.

28. Olivier M, Hollstein M, Hainaut P (2010) TP53 mutations in human cancers: origins, consequences, and clinical use. Cold Spring Harb Perspect Biol 2(1): 172-175.

29. Andrew CRM, Angelo MF, Alison LC, Tina H, Olivier M, et al. (2002) Integrating mutation data and structural analysis of the $p 53$ tumour suppressor protein. Human Mutation 19(2): 149-164.

30. Girardini JE, Napoli M, Piazza S, Rustighi A, Marotta C, et al. (2011) A Pin1/mutant $p 53$ axis promotes aggressiveness in breast cancer. Cancer Cell 20(1): 79-91.

31. Farrell AS, Pelz C, Wang X, Daniel CJ, Wang Z, et al. (2013) Pin1 regulates the dynamics of c-Myc DNA binding to facilitate gene regulation and oncogenesis. Xenbase, 33(15): 2930-2949.

32. Asghar U, Witkiewicz AK, Turner NC, Knudsen ES (2015) The history and future of targeting cyclin-dependent kinases in cancer therapy. Nat Rev Drug Discov 14(2): 130-146.

33. Sheppard KE, McArthur GA (2013) The cell-cycle regulator CDK4: An emerging therapeutic target in melanoma. Clin Cancer Res 19: 53205328 .

34. Fei Q Shang K, Zhang J, Chuai S, Kong D, et al. (2015) Histone methyltransferase SETDB1 regulates liver cancer cell growth through methylation of $p 53$. Nat Commun 6: 8651.

35. Robles AI, Harris CC (2010) Clinical outcomes and correlates of TP53 mutations and cancer. Cold Spring Harb Perspect Biol 2(3): 20-27.

36. Stern MC, Umbach DM, Yu MC, London SJ, Zhang W, et al. (2001) Hepatitis B, Aflatoxin B1 and p53 codon 249 mutation in hepatocellular carcinoma from guangxi, people republic of China and a meta-analysis of existing studies. Cancer Epidemiol Biomarkers Prev 10(6): 617-625.

37. Kirk G, Camus RA, Mendy M, Goedert JJ, Merle P, et al. (2000) Ser-249 p53 Mutations in plasma DNA of patients with hepatolocellular carcinoma from the Gambia. J Natl Cancer Inst 92: 148-153.

38. Ozturk M (1994) p53 Mutations in nonmalignant human liver: Fingerprints of aflatoxins. Hepatology 21(2): 600-601.

39. Senerwa DM, Sirma AJ, Mtimet N, Kangethe EK, Grace D (2016) Prevalence of aflatoxin in feeds and cow milk from five counties in Kenya. African J Food, Agri Nutri Develop 16(3): 11004-11021. 